\title{
Aneddoti e storie di una vita aliena
}

Anecdotes and History of an Alien Life

\section{Alessandro Ferraro}

\section{(e) OpenEdition}

\section{Journals}

Electronic version

URL: http://journals.openedition.org/imagesrevues/4119

DOI: 10.4000/imagesrevues.4119

ISSN: 1778-3801

\section{Publisher:}

Centre d'Histoire et Théorie des Arts, Groupe d'Anthropologie Historique de l'Occident Médiéval, Laboratoire d'Anthropologie Sociale, UMR 8210 Anthropologie et Histoire des Mondes Antiques

\section{Electronic reference}

Alessandro Ferraro, "Aneddoti e storie di una vita aliena", Images Re-vues [Online], 14 | 2017, Online since 03 November 2017, connection on 30 January 2021. URL: http://journals.openedition.org/ imagesrevues/4119; DOl: https://doi.org/10.4000/imagesrevues.4119

This text was automatically generated on 30 January 2021.

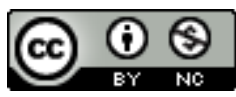

Images Re-vues est mise à disposition selon les termes de la Licence Creative Commons Attribution -

Pas d'Utilisation Commerciale 4.0 International. 


\section{Aneddoti e storie di una vita aliena}

Anecdotes and History of an Alien Life

\section{Alessandro Ferraro}

“Un extraterrestre disse ad una donna Sumera: nostro figlio sarà il primo artista della terra, poi verrà il secondo, poi il terzo. Il primo e il terzo saranno un'opera immortale. L'opera sarà identica a questa che ora stai vedendo. Nell'universo esistono moltissime opere come questa. Questa è l'opera"1. 
1 Al momento della morte di Gino de Dominicis, avvenuta per infarto nel 1998 nella sua casa romana, sono stati ritrovati vari oggetti nel suo studio: statuette sumere, lavori incompiuti, pietre e talismani, una complessa costellazione di pianeti stagliata su un intenso blu cremisi e sul tavolo da lavoro, una copia de Il pianeta degli dei di Zecharia Sitchin ${ }^{2}$. De

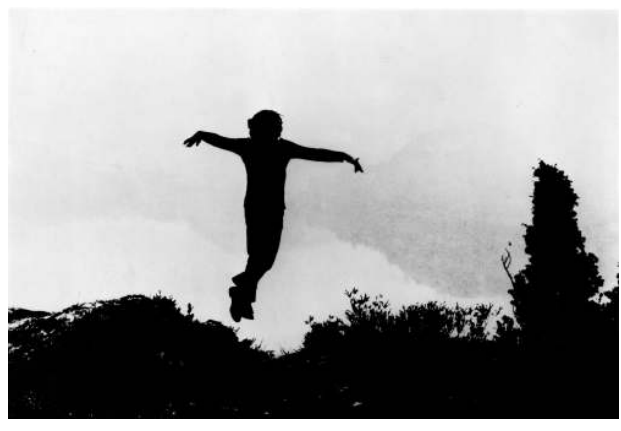
Dominicis possedeva un'edizione particolare del libro: lui stesso è intervenuto sulla copertina scarabocchiandola con alcuni simboli sumeri che alludono all'immortalità dell'anima. Più volte de Dominicis ritornerà sul testo, corredandolo di annotazioni e considerazioni varie; l'intera sua produzione degli anni Novanta, e non solo, è dedicata a tali interessi parascientifici.

Fig. 1

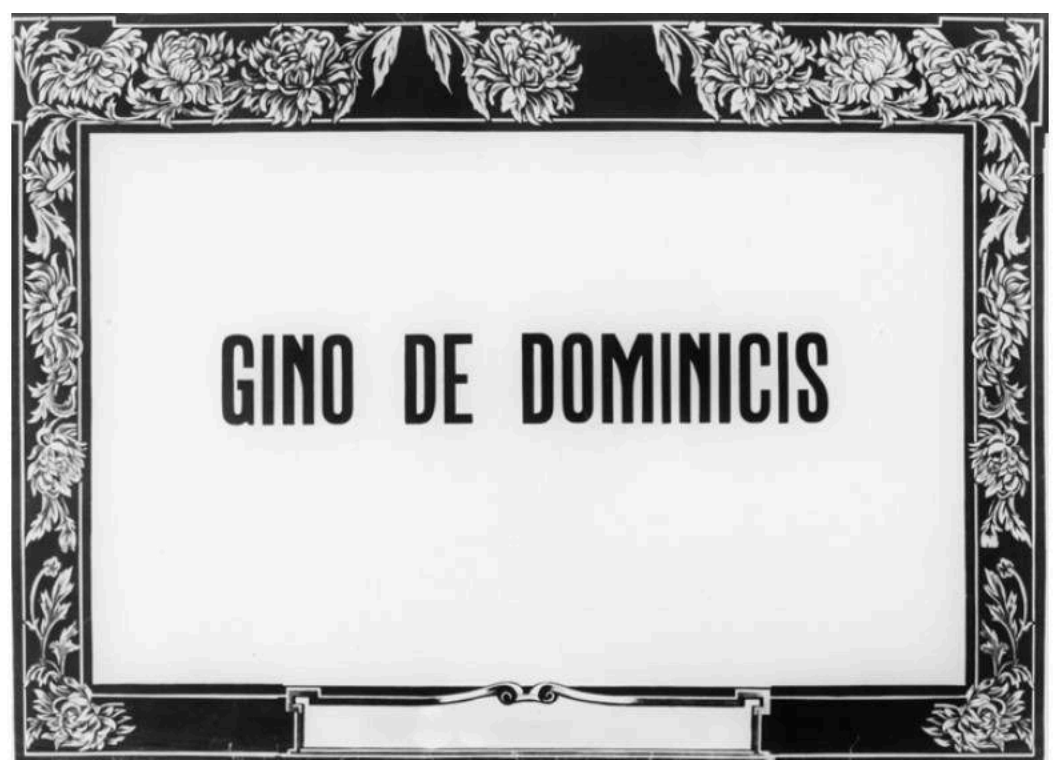

Gino de Dominicis, Manifesto mortuario, 1969, Stampa tipografica su carta, 70 x $100 \mathrm{~cm}$.

Collection privée : http ://www.fabiosargentini.it/mostre_performance_teatro/gino_de_dominicis

2 Al fine di documentarne la grande produzione, benché abbia sempre dichiarato di non voler alcun tipo di catalogo personale, di recente sono stati pubblicati due cataloghi comprensivi dell'opera omnia, utili per comprenderne l'importanza e l'influenza sulle nuove generazioni di artisti.

3 Per analizzare Gino de Dominicis all'interno del contesto italiano e per chiarirne gli interessi verso la para-scienza e le forme di vita aliene manifestati nel suo ultimo periodo di produzione, è utile illustrare gli aneddoti che hanno contribuito a creare un'immagine misteriosa e a tratti grottesca dell'artista. In molti lo ricordano come uno degli artisti più restii nel rilasciare interviste, tanto da tramandare la sua figura ai posteri come un non meglio precisato "artista concettuale".

4 I lavori dell'artista (Ancona, 1947) si possono ascrivere ad una tendenza in voga tra gli anni Sessanta e Settanta in Italia, ovvero lo studio del rapporto tra opera e 
comportamento e la messa in crisi di tale dialettica. La sua produzione, tradizionalmente, viene suddivisa in due periodi: il primo, sopracitato, si estende fino al finire degli anni Settanta, quando comincia il secondo in cui abbandona le sperimentazioni legate al comportamento e alla performance, per dedicarsi alla pittura e all'elaborazione di mitologie personali. La sua parziale storicizzazione, dovuta allo scarso interesse dell'artista a rilasciare interviste o a fornire materiale documentario, ha creato un fiorire aneddotico che se da un lato ha contribuito ad aprire dibattiti e nuove questioni, dall'altro ha complicato la ricerca storico-artistica ${ }^{3}$.

Fig. 2

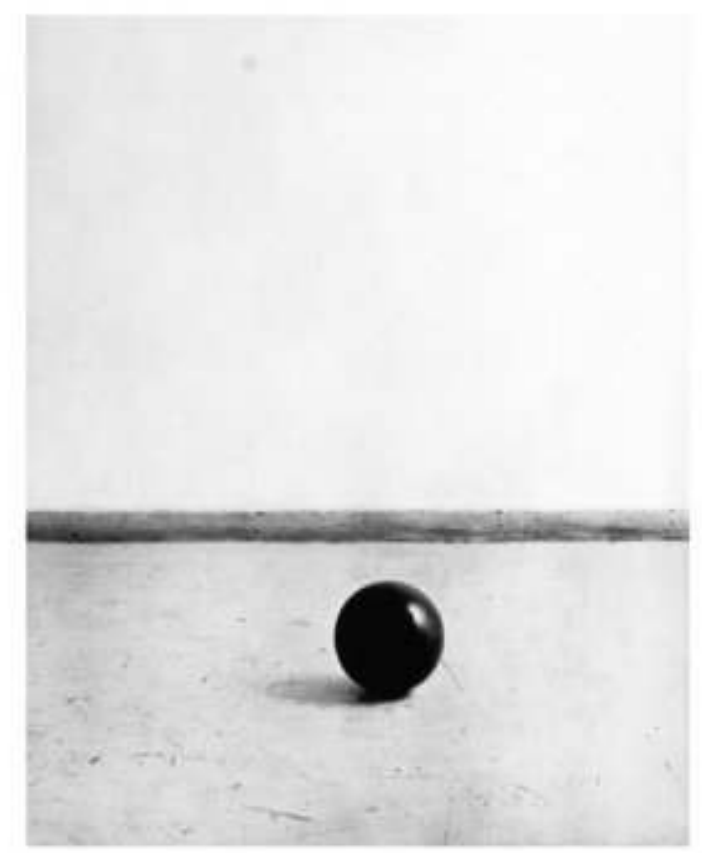

Gino de Dominicis, Palla di gomma caduta da due metri nell'attimo immediatamente precedente il rimbalzo, mixed media, dimensioni variabili, 1969

Courtesy Claudio Abate : http ://www.galleriailponte.com/content/images/ d8429bfde0576aac4a18303d81e55660.jpg

5 Al contrario di quanto facessero molti artisti a lui coevi, basti pensare ai gruppi legati all'Arte Povera o alla Transavanguardia, ha sempre evitato l'assimilazione a gruppi e a sodalizi artistici, fuggendo sia l'approvazione della critica sia quella del pubblico. Quanto più la riflessione dell'arte povera e delle neo-avanguardie internazionali si rivolgeva all'analisi dello spazio e dell'ambiente come elementi determinanti della definizione di luogo e del linguaggio, tanto più la scelta di rifugiarsi in sé, appartato, alla ricerca dell'immortalità, lo rendeva realmente antitetico rispetto al clima artistico che andava per la maggiore in quegli anni. Ma forse più che antitetico, lo si potrebbe definire alieno, in quanto straniero al contesto e, da questa sua condizione, si può desumere il suo interesse verso l'esistenza di forme di vita aliene. Vediamo allora di analizzare il nesso tra il suo sentirsi "alieno" dal sistema dell'arte contemporanea italiana e il suo forte interesse verso le forme di vita extraterrestri. 
6 La sua prima importante personale risale al 1969, presso la galleria romana L'Attico, gestita da Fabio Sargentini. Questa mostra può essere presa a modello per comprendere la sua capacità artistica e la sua inventiva, oltre alle sue aspirazioni artistiche future. Pur facendo parte dell'entourage di artisti che gravitavano attorno alla storica galleria romana, ha sempre cercato di mantenere le distanze, sia dalle frequentazioni sia dalla poetica artistica. L'Attico, in Via Beccaria, era un ex garage: l'ambiente in cui per la prima volta espone l'artista anconetano si popola di molte sue opere, oggi solo parzialmente documentate, data la volontà dell'artista di proibire ogni forma documentaria o pubblicitaria. Gran parte di queste illustrano un suo modo di porsi non convenzionale nei confronti dei dogmi della scienza: Palla di gomma (caduta da due metri) nell'attimo immediatamente precedente il rimbalzo (Fig. 2), Cubo invisibile, Aspettativa casuale di movimento molecolare generale in una sola direzione tale da generare un movimento spontaneo del materiale, il suo stesso Necrologio (Fig. 1), Secchio con acqua sospeso da terra con il gancio di una catena che fa presa sull'acqua, Due verifiche di invisibilità, Equilibrio e Poltrona per un viaggio nello spazio. (Fig 3-4-5)

Fig. 3

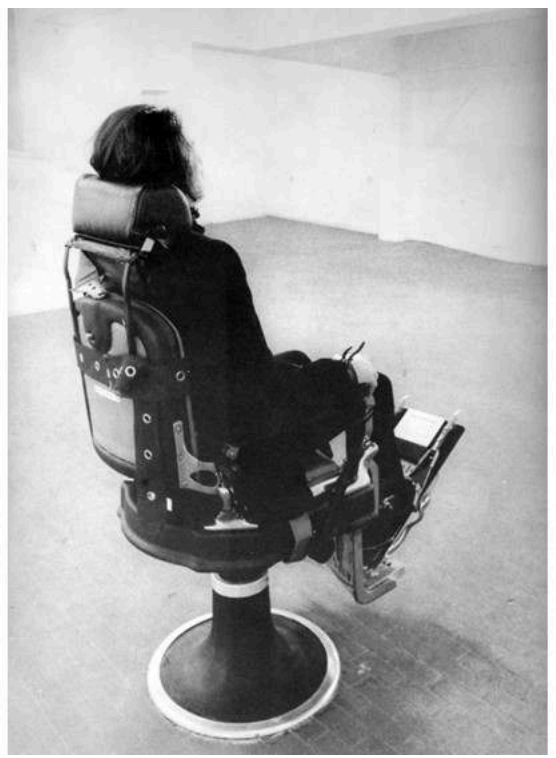

Gino de Dominicis, Poltrona per viaggiare nello spazio, mixed media, dimensioni variabili. Courtesy Gallerie l'Attico : http ://www.fabiosargentini.it/mostre_performance_teatro/ gino_de_dominicis

7 Esse si limitano a descrivere se stesse, come fossero una tautologia; hanno sfumature a tratti paradossali, a metà tra la riflessione metalinguistica e l'impossibile realizzazione di quanto prescrivono nel loro stesso titolo. De Dominicis le ha a più riprese definite come "verifiche di realtà". I suoi conoscenti sono sempre stati sorpresi dal fatto che considerasse le sue opere come entità viventi, dotate di una volontà propria: la tradizione aneddotica si confonde in un misto di stupore e fede che non fa altro che creare ulteriori aneddoti su di lui ${ }^{4}$. Nell'arco di trent'anni la sua posizione verso il paradosso non è mai cambiata, semmai si è rafforzata nel tempo, nutrendosi a sua volta di inconciliabili posizioni estetiche, a tratti retrograde e reazionarie ${ }^{5}$. 
Fig. 4

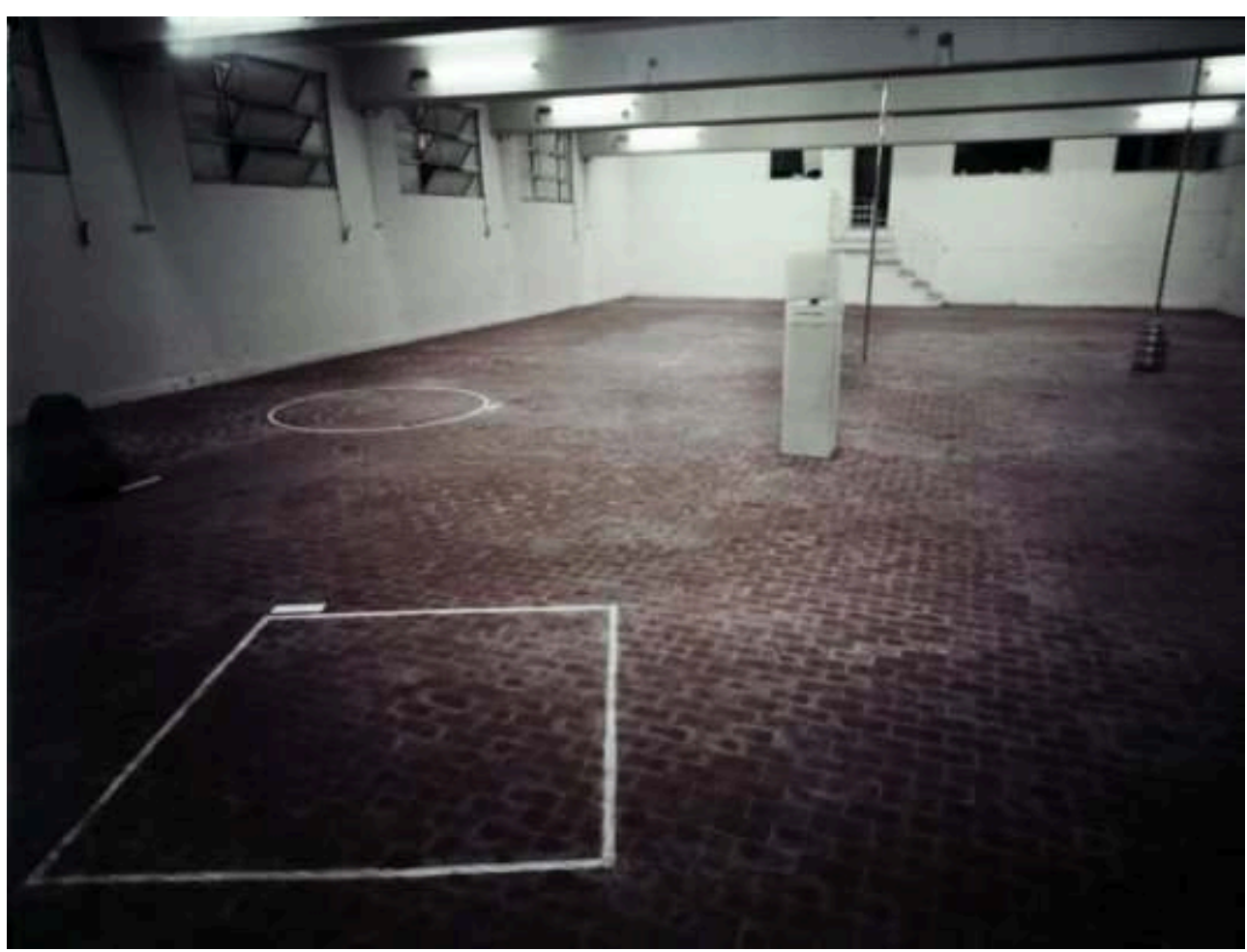

Gino de Dominicis, Installation view, Galleria l'Attico, 1969.

Courtesy Claudio Abate : http ://www.galleriailponte.com/index.php ?it_abate-per-de-dominicis

8 Si può affermare che le opere di de Dominicis, in occasione della sua prima personale, sostenessero che tutta la riflessione sullo spazio artistico, e più in generale sulla realtà, portata avanti dall'arte povera e dal minimalismo, fosse una menzogna. ${ }^{6}$ Uno dei rilievi critici più attenti dell'epoca è una recensione di Renato Barilli di questa personale: il critico si sofferma proprio su quest'attitudine del mostrare e allo stesso tempo dimostrare qualcosa attraverso l'opera d'arte. Il critico scrive di "abilità oratorie" dell'opera d'arte quasi fosse un'entità a se stante, capace di vivere un tempo e uno spazio propri. ${ }^{7}$ Toni simili vengono usati per le sue mostre a venire, facendo emergere sempre più una condizione di sospensione temporale delle sue opere. Quest'ultima caratteristica sposa anche l'idea di sospendere il giudizio e la razionalità logica dall'effetto "oratorio" delle sue opere: queste appaiono come rivelazioni di una realtà personale e soggettiva ${ }^{8}$.

9 Ai fini di questa trattazione è importante osservare come l'artista abbia sviluppato i presupposti sulla riflessione dell'arte nello spazio in maniera estremamente letterale, e in parte ironica: il suo interesse per lo "spazio" è dimostrato da Poltrona per un viaggio nello spazio nella mostra del 1969. La sedia, ricorda Barilli, assomiglia a una seduta da barbiere; l'artista unisce così la strampalata idea di un viaggio nello spazio alla quotidianità suggerita dalla poltrona. Tuttavia, la tematica relativa allo spazio non è strumentale; l'opera stessa rappresenta un tentativo impossibile di viaggiare da fermi, nel tempo e nello spazio, attraverso gli universi. L'idea di un viaggio ipotetico si tramuta in tentativo di formalizzare un concetto impossibile, un ridicolo paradosso che trova coerenza logica esclusivamente all'interno dell'universo singolare dell'artista. 
Fig. 5

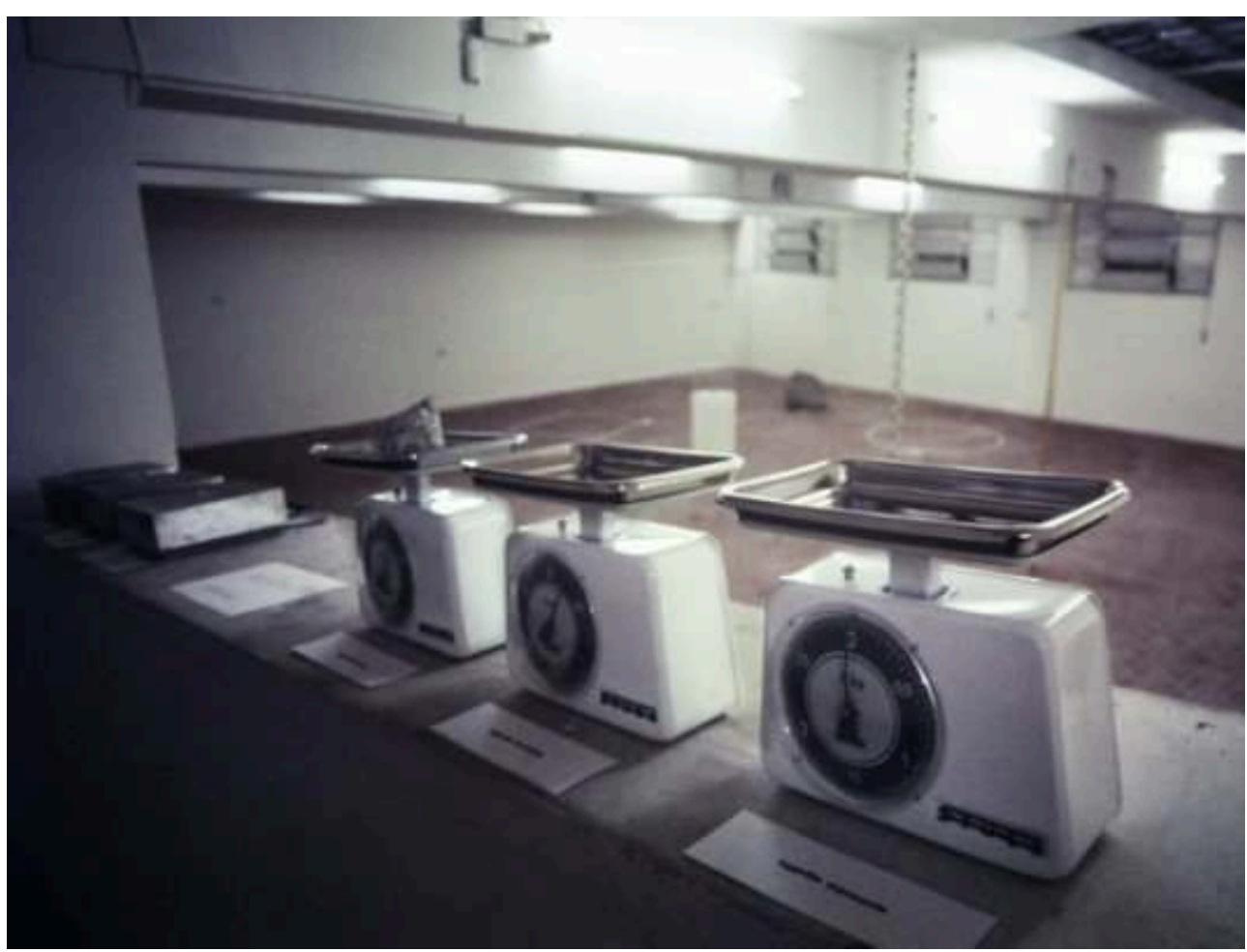

Gino de Dominicis, Installation view, Galerie L'Attico, 1969.

Courtesy Claudio Abate : http ://www.galleriailponte.com/index.php ?it_abate-per-de-dominicis

10 In quegli anni, Gino de Dominicis mette in crisi il rapporto tra finzionalità artistica $\mathrm{e}$ realtà biologica perché decide di seguire una metodologia legata alla presentazione "tale e quale" dell'opera: i titoli delle opere non sono nient'altro che tautologie apparentemente prive di significato che rimandano all'azione che l'artista ha scelto di dare loro'. La critica nei confronti dell'arte concettuale italiana si può intuire dal funzionamento dei suoi lavori di questo periodo: in Zodiaco (1970) (Fig 6), un'opera in cui la fascinazione verso lo spazio e l'universo è evidente, progetta una riproposizione tale e quale dei simboli zodiacali sostituiti con persone, oggetti e animali viventi, realizzando un tableau vivant: gli interpreti dei segni zodiacali sostituiscono il concetto di simbolo attraverso l'autopresentazione. In una delle sue rare e ultime interviste, l'artista ricollegherà la realizzazione dello Zodiaco ad una suggestione proveniente dall'arte e dalla conoscenza dei Sumeri, da lui stesso tanto approfonditi, i quali, stando all'artista, consideravano in maniera più sacrale rispetto alla contemporaneità il valore dello zodiaco ${ }^{10}$.

La tanto celebrata smaterializzazione dell'oggetto da parte degli artisti concettuali, non solo italiani, trova in Gino de Dominicis una controparte attiva perché lui stesso mette in crisi attraverso la presentazione tale e quale dell'oggetto-opera l'idealismo e i concetti linguistici su cui il concettuale stesso si fonda. Laddove in de Dominicis è presente una qualsivoglia forma di smaterializzazione, si pensi ai Quadrati invisibili semplici righe di scotch bianco tracciate sul pavimento ad indicare la scomparsa dell'oggetto come in una scena del crimine -, è sempre presente una controparte attiva che mette in ridicolo la stessa idea di concettualità. Non solo, le sue stesse opere, intese 
come "verifiche di realtà", sono supposte prove fisiche che vanno in contrasto con le leggi scientifiche, spesso volutamente banalizzate e ridicolizzate dall'artista.

\title{
Contro la scienza, contro il tempo: per un vivere ultraterreno
}

\begin{abstract}
"Cara...Io penso che le cose non esistano. Un bicchiere, un uomo, una gallina, ad esempio, non sono veramente un bicchiere, un uomo, una gallina, ma solo la verifica della possibilità di esistenza di un bicchiere, di un uomo, di una gallina. Per esistere veramente le cose dovrebbero essere immortali, eterne, solo così non sarebbero solo delle verifiche di certe possibilità, ma veramente cose"11.
\end{abstract}

Anti-scientismo e ricerca dell'immortalità sono alcuni dei punti cardine della ricerca di Gino de Dominicis: il suo tardo interesse verso le forme di vita extraterrestri, come ribadito dalla sua fascinazione verso la controversa figura di Zecharia Sitchin, risulta essere una naturale conseguenza del suo pensiero. La ragione per cui de Dominicis abbia escluso la scienza teorica dai propri interessi e abbia preferito dedicarsi alla pseudoscienza è legata alla sua personalità estrosa; i motivi del porre in ridicolo $i$ dettami scientifici, immaginando altri mondi, altri esseri senzienti capaci di interagire con l'uomo, consistono nel tentativo di rendere non interpretabili le proprie opere e di conferire loro un'aura misteriosa.

De Dominicis accosta un lato giocoso, a tratti ironico, ad una pretesa di verità scientifica garantita dalle proprie stesse opere: il porre tra le virgolette e la sospensione del giudizio su un atto estetico assumono a tratti una valenza affettuosa nei confronti delle proprie opere, trattate, come ricordato, come parti del sé. Emblematico in questo caso è l'opera Tentativo di volo, un breve video che riprende i suoi innumerevoli tentativi nel cercare di spiccare il volo da uno sperone roccioso. Ricorda Germano Celant che Tentativo di volo prevedeva un'iterazione infinita del tentativo di spiccare il volo (Fig. 7): secondo l'artista infatti i suoi pronipoti, perseguendo una simile azione, prima o poi si sarebbero evoluti passando ad uno stadio superiore dell'esistenza, riuscendo infine a spiccare il volo, coronando il sogno del loro $\mathrm{avo}^{12}$. Un simile discorso può essere fatto per Tentativo di far formare dei quadrati invece che dei cerchi intorno ad un sasso che cade dall'acqua (1969), video-performance nel quale l'artista compie esattamente quanto affermato nel titolo. Nelle due opere sopracitate si evince una connotazione comportamentale decisiva per comprendere le sue opere a venire: la ricerca del paradosso, come realtà ultima della vita umana, è solo il primo passo che il pubblico e le persone dovrebbero compiere nel cercare di raggiungere una non meglio precisata immortalità, capace di porre fine al paradosso della vita umana.

I motivi per cui scrive una Lettera sull'immortalità si comprendono bene alla luce delle sue considerazioni su uomo, storia e scienza; scritta inizialmente nel gennaio 1970, viene seguita da un'altra versione pubblicata nell'aprile dello stesso anno, parzialmente simile, che tuttavia non modifica il contenuto principale, ovvero, una giustificazione dell'inesistenza delle cose attraverso la ricerca costante del motivo ultimo dell'esistenza. L'incipit della lettera si rivolge ad un'ipotetica donna, presumibilmente anche all'intero genere femminile, inteso come controparte maschile; in seguito passa in rassegna ogni sua convinzione in merito al tema della realtà e dell'inesistenza o meno della stessa. L'incipit, nella sua icasticità, ammette anche un gravoso fallimento: l'impossibilità di trovare definitivamente l'immortalità per mano umana. Tuttavia il 
tono autocommiseratorio viene sostituito dalla prova tangibile del fallimento, dall'impossibilità per le cose terrene di esistere realmente, di essere soltanto "cose" sottoposte al processo degradante del tempo. Nel pensiero dell'artista si possono osservare numerose contraddizioni logiche le cui implicazioni però non si estendono all'applicazioni di ideologie o mode artistiche: per de Dominicis sembra esistere un'unica realtà, quella immortale. Egli applica un processo di esclusione rispetto alla contemporaneità, si sottrae al tempo: la lettera diventa un vero e proprio manifesto che, a differenza dell'arte del suo tempo, non si basa su teorie linguistiche care agli artisti concettuali, ma parla direttamente alla vita e alle cose con una sincerità e una schiettezza tanto spiazzanti da risultare paradossali.

Italo Tomassoni ricorda come de Dominicis entrò in un acceso dibattito con lo scienziato A. M. Liguori in merito alla teoria dell'entropia e della termodinamica. L'artista sosteneva come fosse possibile arrivare a concepire l'immortalità e a viverla sul proprio corpo, a dispetto della società scientifica e della teoria entropica ${ }^{13}$. Ebbe inoltre modo di elaborare, grazie al supporto di Franco Rustichelli, una teoria pseudoscientifica basata su due tipologie di immortalità, una dinamica, l'altra costante. (Fig. 8) Non è la prima opera che de Dominicis utilizza per andare contro i dogmi scientifico-matematici: nel 1969 realizza 1+0=0, un semplice disegno che intende confutare le basilari convenzioni matematiche ${ }^{14}$. Nel 1980 realizza presso la Galleria Pio Monti di Roma un Lampadario antientropico, consistente in un grande lucernaio interamente fasciato con tele da lui dipinte; l'opera dimostrava come l'energia artistica si immagazzinasse all'interno del lampadario concentrando l'energia dello sforzo $\operatorname{artistico}^{15}$.

L'opera che segna inequivocabilmente il successo di de Dominicis - e che approfondisce il suo interesse verso l'immortalità come idea biologica extraterrestre - è Seconda soluzione di immortalità (l'universo è immobile), allestita in occasione della Biennale di Venezia del 1972. Eleonora Charans ha ricreato, tra le difficoltà che le disposizioni di de Dominicis hanno comportato, l'aspetto della sala e dell'installazione; all'interno delle stanze del padiglione centrale della Biennale, una persona, Paolo Rosa, affetto da sindrome di Down, sta seduto su una sedia in un angolo; accanto alcuni lavori precedenti come Palla e Quadrato invisibile, poi ancora simboli stampati su carta, alcuni di questi raffiguranti svastiche, e altri elementi di matrice ideologica (Fig. 9).

L'idea di proporre una soluzione teorica al problema dell'immortalità è di per sé una trovata eroicomica; la presentazione stessa dell'opera, fin dal titolo, presenta gli stilemi tipici del personaggio "Gino de Dominicis": letteralità e certezza del fallimento dell'esperimento sono i termini entro cui l'opera agisce ${ }^{16}$.

Nella Seconda soluzione di immortalità (l'universo è immobile), Paolo Rosa è compiutamente immortale perché non può pensare alla morte. Infatti chi è affetto dalla sindrome di Down soffre di un deficit cognitivo tale da renderlo alieno alla realtà. Nella fotografia Paolo Rosa è seduto immobile sulla sedia e sembra guardare il pubblico: il suo è "un vedere che non si traduce in sapere"17. Il suo mutismo sembra quasi ad aggredire lo spettatore. Paolo Rosa, secondo de Dominicis, risulta essere un'entità sovrasensibile, parallela, che percepisce una realtà radicalmente differente dalla nostra e invita, con il suo sguardo, a tratti bonario, a riflettere sul fatto che "l'uomo è un'incognita nello spazio-tempo"18. Oltre ad insistere su un differente concetto di tempo, l'opera si basa su un diverso tipo di spazialità: l'artista infatti ha sempre concepito quest'ultima come una contingenza necessaria al tempo, non viceversa, come gran parte degli artisti legati 
al modernismo, basti pensare a Lucio Fontana, o a tutta la generazione successiva di artisti minimal americani ${ }^{19}$.

Il sentirsi alieno dal mondo di Paolo Rosa verrà ripreso da de Dominicis in altre opere: tra queste, Paolo Rosa a Venezia nel 1972 (1987), che mostra il suo profilo di spalle, intento a contemplare un pianeta distante nell'universo. In L'antieffimero (1985), è presente un profilo umano realizzato a foglia d'oro, che si staglia su un fondale monocromo, anch'esso intento a scrutare mondi lontani. Ambedue le opere non sono mai state esposte al pubblico. L'ultima opera che si rifà idealmente alla figura di un uomo che scruta lo spazio profondo è Ritratto di Carmen D. V. (1998), una delle ultime opere di de Dominicis, esposta in occasione della mostra Sicinimod Ed presso la Galleria Mazzoni. Il fatto che abbia scelto di esporla permette di scrivere un ideale percorso iniziato nel 1972 e terminato nel 1998 riguardante l'uomo in dialettica con lo spazio ${ }^{20}$.

De Dominicis, inconsciamente, si affida a quel modello teorico tracciato da Susan Sontag in Notes on camp, muovendosi concettualmente tra utopia modernista - in contrasto con la scienza - e nostalgia verso culture lontane e primordiali. Sontag ha descritto la produzione culturale degli anni Sessanta, e grosso modo anche quella a venire, come un momento dialettico in tensione tra queste due forze ${ }^{21}$. Da un lato lo sguardo dell'artista è nostalgico, ma al contempo è utopico perché consapevole del proprio fallimento in quanto essere umano; tuttavia, non per questo si astiene dal continuare a produrre "verifiche di realtà" e di cercare di essere immortale, in aperto scontro con il metodo scientifico.

\title{
Gilgamesh e le presenze ultraterrene
}

\author{
"MM: In alcune sue opere vi sono figure che sembrano dei veri e propri \\ extraterrestri. Quindi lei crede all'esistenza di civiltà extraterrestri? \\ GDD: $\mathrm{Si}$ \\ MM: Gli antichi Sumeri raccontano nelle loro scritture di essere stati iniziati alla \\ cultura e di aver appreso tutte le loro conoscenze da una civiltà proveniente dallo \\ spazio. Lei pensa che tutto ciò sia vero? \\ GDD: $\mathrm{Si}^{\prime 2}$
}

21 Il continuo ritagliarsi, da parte dell'artista, un profilo personale dal sistema-mondo in cui ha operato è stato uno dei punti saldi della sua estetica: essere alieno, di fronte a tutto e tutti, abitare uno spazio inconsistente, a tratti irreale e irrintracciabile, vivere un tempo passato, fatto di contraddizioni storiche e affascinato da mitologie antiche sono vere e proprie metodologie artistiche. Il fatto di vivere appartato, lontano dalle luci dello spettacolo, chiarifica la scelta di inventarsi delle mitologie personali. Non a caso, dopo la stagione legata alla contestazione del concettuale, de Dominicis si affida alla pittura. È stato uno dei pochi artisti a non percepire in maniera violenta questo distacco procedurale in quanto i suoi tableaux vivants costituiscono la diretta conseguenza delle sue teorie.

All'inizio degli anni Ottanta la produzione pittorica di de Dominicis si popola di una miriade di simboli arcaici, sumeri, citazioni da epopee indiane, fino a supposizioni, sostenute fino alla morte, di forme di esistenza aliena. Nelle tele degli anni Ottanta e Novanta l'artista anconetano ha ampliato la propria ricerca artistica frequentando letterature e mitologie orientali e sumere: l'ossessione per la ricerca dell'immortalità viene fatta propria per emulare tali culture. Tra gli aspetti più tipici di tale fascinazione è il suo interesse verso forme di bellezze arcaiche - come testimoniano le tele del suo 
ultimo periodo (In principio era l'immagine, 1980; Opera Ubiqua, 1993;): una caratteristica, questa, che trova nella coincidenza tra ossessione verso una bellezza primordiale e la ricerca dell'immortalità la principale ragion d'essere. Tra le varie fonti che l'artista consultava vi era senz'altro una cospicua quantità di testi pseudo-scientifici, molto in voga a partire dagli anni Settanta, diretta conseguenza delle filosofie new-age nella cultura occidentale.

Il pianeta degli dei di Zecharia Sitchin, secondo Italo Tomassoni, lo accompagnava sempre in ogni mostra in cui si recava, tanto da essere un vero e proprio testo sacro per l'artista. Sitchin diventa immediatamente famoso negli ambienti para-scientifici a seguito della pubblicazione de Il pianeta degli Dei, a cui seguiranno molti altri testi ad esso derivati ${ }^{23}$ : le teorie dello studioso traggono origine da concetti inventati che cercano di trovare una giustificazione razionale nella mancanza di fonti storiche. Da tale vuoto storico, le teorie di Sitchin trovano applicazione soprattutto nella paraarcheologia e nei miti di fondazione terrestre, legati a doppio filo, secondo lo studioso, ad alieni, i quali avrebbero donato tecnologie avanguardistiche ai Sumeri e alle prime popolazioni terrestri. Il fervore di de Dominicis nell'applicare e nel produrre opere che rimandano alle teorie di Sitchin è senz'altro sintomo di un adesione sincera alle citate teorie parascientifiche: la sua stessa fiducia in idee simili assume connotati peculiari, come se de Dominicis si considerasse erede di quell'arcaica storia culturale.

Uno dei motivi principali per cui è rimasto affascinato dalla cultura sumera, oltre all'influenza di Sitchin, è la figura di Gilgamesh, unico eroe vivente in grado di raggiungere l'immortalità. Come ripetuto, non sono secondari gli aneddoti che gravitavano attorno all'artista in quegli anni; Gino de Dominicis si considerava un semiDio, al pari di Gilgamesh, unico tra i semidei raccontati dai miti antichi ad essere al contempo artista, architetto, regnante e semi-immortale. Lo spettatore quindi, stando ai dettami dell'artista, non può che esperire un elemento misterico e iniziatico attorno alle sue opere; per tali motivi infatti le sue opere sono da considerarsi come rivelazioni filosofico-antropologiche. La scelta dei Sumeri come mitologia "sorella" è indicativa poiché loro credevano fermamente nell'immortalità dei corpi durante la vita terrena. Di conseguenza, la ricerca della stessa aveva connotati eroici, tanto che l'artista anconetano li fa propri al punto da investire la ricerca dell'immortalità di connotazioni artistiche. Esistono le fotografie di un'opera dell'artista, andata in seguito distrutta, la cui documentazione è stata attentamente ricostruita da Italo Tomassoni, che testimonia come Roma fosse stata fondata dagli stessi sumeri, centinaia di secoli prima $^{24}$.

Secondo de Dominicis, l'artista per eccellenza è un essere semidivino, al pari di Gilgamesh, capace di ristabilire l'ordine temporale e spaziale dell'eternità. L'istantaneità di tale rivelazione si ricollega alla questione concernente la fotografia e la sua capacità di fissare l'attimo, eternarlo nonostante la durata effimera del supporto fotografico. Esistono pochi esperimenti dell'artista alle prese con la fotografia, ma quasi tutti sono decisamente rilevanti ai fini di questo studio. Le prime opere della serie sulla mitologia antica consistevano in alcuni lavori realizzati su schermi trasparenti che, oltre a recare i profili di Gilgamesh e Urvasi, ninfa indiana che presiedeva il culto della bellezza, permettevano di guardare oltre il supporto stesso; sono stati installati per la prima volta alla Galleria Sprovieri di Roma in Piazza del Popolo, nel 1983. Benché siano stati esposti circa tre anni dopo la prima opera della serie Urvasi e Gilgamesh, i lavori sopracitati testimoniano una ricerca decisamente sui generis per l'artista anconetano. La 
sua scelta di intervenire direttamente sulla fotografia si inserisce nella volontà di riscrivere la realtà, di popolarla delle sue visioni personali; la realtà di Piazza del Popolo si costella così di presenze ultraterrene, invisibili ai più se non attraverso lo stratagemma di de Dominicis.

Tra le prime rappresentazioni della serie Urvasi e Gilgamesh si ricorda il lavoro del 1980 (Fig 10), nel quale, tra i due profili si scorge uno strano panorama con una piramide sullo sfondo e, secondo l'interpretazione fornita da Maurizio Calvesi, un Ufo che domina la scena. La serie di Urvasi e Gilgamesh lo terrà occupato per tutta la vita (Fig 11); parallelamente non smette di esplorare nuove forme di rappresentazione del concetto di divinità, utilizzando principalmente la pittura, ma anche il disegno. Accanto a tali rappresentazioni occorre precisare la grande importanza che Zecharia Sitchin aveva per le teorie di de Dominicis in merito all'immortalità del corpo e, soprattutto, alla fondazione del pianeta terra per mano di alieni provenienti da un pianeta di un altro sistema solare, il cosiddetto pianeta Nibiru. In una delle sue opere tarde, Pianeti (1987) (Fig. 12), si può addirittura osservare come l'artista anconetano si sia ispirato ad una rappresentazione fatta da Sitchin nel suo libro ${ }^{25}$. Il para-scienziato americano utilizza uno schema planetario che verrà copiato in ogni suo dettaglio dallo stesso de Domincis: nel disegno si può osservare il sistema solare corredato dagli undici pianeti, con l'aggiunta del supposto "dodicesimo" pianeta, da cui sarebbero provenuti gli alieni che donarono le nuove tecnologie ai terrestri ${ }^{26}$. I cosiddetti Nephilim, oltre a creare la razza umana, l'avrebbero seguita a debita distanza, influenzandola e facendola progredire tecnologicamente con il passare del tempo ${ }^{27}$. L'artista anconetano non solo trae ispirazioni contenutistiche riguardo al rapporto tra sumeri e forme di vita extraterrestre, ma utilizza il testo di Sitchin anche in senso formale: il pannello centrale di Trittico (1993) (Fig. 13), trae diretta ispirazione dal posizionamento interstellare dei pianeti profetizzato dallo pseudo-scienziato americano ${ }^{28}$. Come si osserva in quest'ultima opera, l'artista aggiunge considerazioni personali al "dodicesimo" pianeta, aggiungendo vari altri satelliti attorno allo stesso. Più che una semplice fascinazione estetica, nel caso di de Dominicis l'ispirazione al concetto di forma di vita aliena è basilare per comprendere il motivo del suo fare artistico nei tardi anni Ottanta. L'entrata di tali soggetti a metà tra la rappresentazione mitologia e l'ideazione di mitologie personali non costituisce un semplice accostamento illustrativo, ma una sincera adesione alle loro credenze.

L'intera produzione pittorica di de Dominicis converge sull'importanza di riprodurre soggetti "visualmente" nuovi, originari, al fine di far riflettere lo spettatore sul senso del tempo e della stratificazione della cultura storica: L'artista affermò a più riprese la necessità di un'originalità legata al metodo pittorico ${ }^{29}$. Durante tutta la sua carriera ha scelto di identificare con la contemporaneità, e con i valori che essa sottende, un vero e proprio nemico artistico, capace di affievolire l'afflato produttivo e l'originalità del genio artistico: oltre a essere una precisa scelta estetica, questa assume connotati etici perché l'artista decide di non "inquinare" il presente storico con opere autoreferenziali, che parlano solo di sé nell'oggi, per realizzare opere, invece, che parlano al futuro e al passato ${ }^{30}$. La concezione temporale di de Dominicis, nonostante appaia in gran parte contradditoria, si inserisce all'interno di un modello circolare, basato sull'eterno ritorno e sull'idea che il passato rappresenta la giovinezza, mentre il futuro la vecchiaia ${ }^{31}$. 

iconografico per le opere pittoriche di de Dominicis al fine di comprendere meglio la commistione di temi, tempi e spazi che racconta attraverso i suoi quadri: la grande produzione della serie dedicata a Urvasi e Gilgamesh funge da apripista a tutte le altre incentrate sul connubio tra mitologia e pseudo-scienza. Numerose infatti sono le tele che ricordano esseri extraterrestri, come lui stesso ha palesato nei titoli delle sue opere: la serie delle Regine è emblematica, richiama stilemi arcaicizzanti e gli sfondi si riallacciano ad una rappresentazione bidimensionale, funzionale al carattere di rivelazione dell'opera stessa (Fig. 17). Per quanto riguarda il tema legato al viaggio, temporale e spaziale, emblematica è in questo senso l'opera L'Astronave (Fig. 18), elemento simbolico che compare a più riprese nelle tele dell'artista, di volta in volte reinterpretata anche nella serie di Urvasi e Gilgamesh. Non meno importanti sono le figure della serie dei Nasi, grandi profili astratti che richiamano in parte a figure umane con grandi nasi protrudenti - in taluni casi questi sporgono all'esterno della superficie pittorica - o dei Guerrieri, figure armate di lancia che si stagliano sulla tela, o ancora le Ballerine, figure stilizzate che rimandano alla serie delle Regine. (Fig. 17) È evidente come l'universo, per de Dominicis, sia un campo di sperimentazione in cui i luoghi più reconditi e inesplorati sono popolati da figure e stilemi provenienti dal passato; il concetto di esplorazione pittorica diventa qui un connubio di teorie pseudoscientifiche, immaginazione personale e revisione della realtà. Anziché popolare lo spazio scenografico delle sue tele di elementi legati alla "contemporaneità", egli presenta modelli e soluzioni antiche, che richiamano aspetti della cultura sumera; in gran parte dell'iconografia legata agli extraterrestri è facile osservare una stella a sei punte, richiamo simbolico all'idea di perfezione dell'universo ${ }^{32}$. Nei lavori attinenti al tema è possibile ritrovare nella soluzione stilizzante e nelle figure bianche su fondo oro, un elemento chiave della produzione, tale da poter intenderla come un solo corpus artistico con molti rimandi tra un'opera e l'altra. L'opera Senza Titolo, realizzata in argilla e tempera era stata esposta a Venezia nel 1990, e corredata da un'installazione intitolata La macchina del tempo: consisteva in un grande teschio poggiato su un grande basamento nero con un sedile sporgente collegato all'imponente installazione. Il teschio con lo strano e peculiare naso a punta si richiama a Calamita cosmica (1988), una delle opere più famose dell'artista, consistente in un grande scheletro di circa venti metri, disposto supino sul terreno, con lo stesso curioso naso a punta. Quest'ultimo costituisce un ulteriore rimando alla teoria extraterrestre elaborata dall'artista, come se la sua aspirazione alla ricerca di mondi lontani potesse concretamente modificare la struttura scheletrica umana, ingrandendone lo scheletro e dotandolo di naso osseo, la cui punta affilata fungesse da luogo di contatto tra uomo e alieno.

30 Il caso di Bit Akinu Retrospectiva (1994), una tela che mostra un incontro tra due entità non meglio definite, è emblematico perché fonde impianti figurativi differenti: da un lato c'è un richiamo evidente alla serie Prospettiva Rovesciata (Fig. 20) e a L'appuntamento, 
nelle quali de Dominicis ribalta la prospettiva lineare per fornire un'idea metafisica di infinito ${ }^{33}$. L'opera L'appuntamento (1987), che unisce gli elementi della Prospettiva rovesciata, mette in scena un incontro extraterrestre, essendo la figura e il soggetto pienamente ascrivibili agli stessi modelli di cui sopra. Inoltre nell'opera del 1994 è palese il riferimento alla serie delle Regine, dove un esserino stilizzato compare nella parte alta del quadro nell'atto di scendere verso il fondo stesso del quadro.

Bit Akinu Retrospectiva non è l'unico caso ${ }^{34}$ : si pensi a tutta la serie dei Pianeti, che compare a più riprese in più soggetti differenti, anche tra i vari Urvasi e Gilgamesh. Un discorso simile può essere fatto anche per un'opera intitolata Senza titolo, nel quale compaiono le medesime figure stilizzate in cerchio, a simulare un girotondo tra figure stilizzate. (Fig. 21; Fig. 22). Come si osserva in quest'ultima opera, le fattezze delle figure in primo piano, la loro forte stilizzazione e l'assenza di un vero e proprio sfondo risultano essere un luogo comune della sua produzione artistica degli ultimi anni. ${ }^{35} \mathrm{La}$ tarda produzione artistica di de Dominicis costituisce, nel quadro di una fervida elaborazione di mitologie personali e di un inedito interesse verso forme di vita aliene, un unicum nella storia dell'arte italiana, sia per il modo in cui è stata trattata sia per i rimandi con la sua produzione artistica precedente; i rimandi alle varie mitologie antiche, le fascinazioni verso le pseudo-scienze, e soprattutto il suo interesse verso le forme di vita aliene sono ancora da approfondire e si è lungi dall'essere arrivati ad una formulazione certa sui modi in cui tali culture abbiano concretamente influito sulle opere di de Dominicis; forse, nonostante i problemi legati alla sua personalità, e di conseguenza alla sua collocazione storico-critica nel contesto italiano, l'aspetto più interessante della figura dell'artista anconetano è il tentativo di unire interessi contraddittori per formare un'ideologia artistica per lui coerente, dalla ninfa indiana Urvasi alle astronavi, ai Nephilim e Nibiru, ottenendo così un complesso patchwork di tessuti culturali, mitologie personali e aspirazioni ideali

\section{NOTES}

1. La citazione proviene da un testo manoscritto a biro proveniente dall'Archivio dell'Associazione Gino de Dominicis. La frase è stata riportata in calce nel catalogo della mostra Gino de Dominicis. L'immortale curata da Achille Bonito Oliva nel 2010 presso il MAXXI di Roma.

2. La ricostruzione dello studio di Gino de Dominicis si basa sui materiali accuratamente schedati e inventariati da Italo Tomassoni, proposti per la prima volta al pubblico nel suo stesso catalogo.

3. In occasione della mostra Sicinimod Ed presso la galleria Mazzoli di Modena (1998), viene pubblicato il suo unico catalogo da lui riconosciuto con il titolo SICINIMOD ED, un evidente palindromo del cognome dell'artista; il catalogo mostra alcune fotografie che ritraggono l'artista al lavoro nel suo studio di Via San Pantaleo a Roma.

4. G. de DOMINICIS, Le mie opere non vogliono essere esposte alla XLVI Biennale di Venezia, in G. GUERCIO (cur.), De Dominicis. Scritti sull'opera e riflessioni dell'artista, Allemandi, Torino, 2014, pp. 265-266.

5. Non è un mistero la sua ritrosia nei confronti di un'arte politica, molto in voga durante tutti gli anni Settanta; negli anni Novanta non recede dalle sue posizioni, anzi, contesta più volte la 
differenziazione stilistica tra arte tradizionale (pittura, scultura, architettura) rispetto al grande calderone dei mixed media, della videoarte e delle installazioni. Si veda il riferimento alla nota 10.

6. C. TORREALTA, Frasi di Gino de Dominicis 1969-1999, in G. Guercio (cur.), op. cit., p. 286.

7. R. BARILLI, «Le persuasioni», NAC, maggio 1970, p. 12

8. F. VINCITORIO, «Gino de Dominicis», NAC, gennaio 1971 pp. 27-28.

9. Questa soluzione originale, che se rimanda in parte al concetto di theatricality trattato da Michael Fried, non svilisce il senso contenutistico dell'opera, ma amplifica il rapporto dello spettatore con la realtà a cui de Dominicis intende farlo partecipare.

10. D. TROBADORI, Promemoria di fine secolo, in G. Guercio (cur.), op. cit., p. 281

11. G. de Dominicis, Lettera sull'immortalità - Roma, 10 Aprile 1970, in G. Guercio (cur.), op. cit., p. 251-255.

12. G. CELANT, Gino de Dominicis, in G. Guercio (cur.), op. cit., p. 169

13. I. TOMASsoni, Il caso Gino de Dominicis, in G. Guercio, op. cit, p. 185

14. I. TOMASSONI (dir.), Gino de Dominicis (catalogo ragionato), Skira, Milano, 2011, p. 238

15. Per la documentazione fotografica dell'opera si rimanda a Ibid., p. 307.

16. Il tentativo di fornire una soluzione al problema dell'immortalità era stato preceduto da un altro esperimento, realizzato in occasione della sua personale alla galleria Toselli di Milano nel 1970, coinvolgendo un gatto con la scritta "seconda soluzione di immortalità" su un cartoncino poggiato sul dorso; il gatto veniva posto in un secondo momento in una teca di vetro e, magicamente, il cartoncino scompariva.

17. G. GUERCIO, L'arte non evolve. L'universo immobile di Gino de Dominicis, Johan\&Levi, Milano, 2015, p. 29

18. Ibidem, p.33

19. Per farsi un'idea dei problemi suscitati dall'esposizione si rimanda a E. Charans, Seconda soluzione di immortalità (l'universo è immobile), Scalpendi, Milano, 2012, pp. 25-30 e P. Pasolini, «Il mongoloide alla biennale è il prodotto della sottocultura italiana», Il Tempo, Roma, 25 giugno 1972, p. 10.

20. Per i riferimenti iconografici e fotografici si rimanda a I. Tomassoni, op. cit., p. 499.

21. S. SONTAG, Against interpretation, Picador, New York, 2001, pp. 20-43.

22. M. MIRolla, L'immortalità. Invervista a Gino de Dominicis, in G. Guercio, op. cit., p. 294.

23. Il primo testo di Zecharia Sitchin è pubblicato nel 1976 con il titolo The 12th Planet. Il numero dodici del titolo si riferisce all'esistenza di un possibile altro pianeta nascosto nel nostro sistema solare.

24. I caratteri figurativi dell'opera, riportata nel catalogo di Italo Tomassoni, rimandano al binomio tra sumeri e entità extraterrestri le quali, secondo l'artista, avrebbero contribuito a dare i natali di Roma. Per le uniche immagini dell'opera si rimanda a I. Tomassoni, op. cit., p. 399.

25. Z. SITCHIN, Il pianeta degli dei, Giunti, Milano, 2003, p. 83.

26. Ibidem, p. 25

27. Ibidem, p.12-34

28. Riferimenti alla mitologia sumera, e non solo, iniziano alla fine degli anni Settanta fino alla sua morte. L'interesse di de Dominicis riguardo all'argomento è tale da creare una commistione e una rilettura personale di tali credenze religiose. L'aspetto peculiare di tale operazione è il suo accostamento tra mitologia sumera e interesse fantascientifico, riscontrabile unicamente negli scritti coevi di Zecharia Sitchin.

29. G. TESTORI, Fiamme degli inferi per l'Uomo-Gufo, (smalto su legno) 1986, in G. Guercio, op. cit., pp. 177-178.

30. Oltre alle sperimentazioni pittoriche di de Dominicis, si annoverano gli altri "ritorni" della pittura, sia del gruppo della Transavanguardia, sia degli Anacronisti. L'uso della pittura da parte 
dell'artista anconetano, sia nelle forme che nei contenuti, ha esiti opposti al successo di critico e di mercato ottenuto dai pittori della Transavanguardia

31. G. GUERCIO, L'arte..., op. cit., p. 72.

32. I. TOMASSONI, op. cit., p. 414.

33. Per i riferimenti fotografici si rimanda a A. BonIto oliva, Gino de Dominicis: l'immortale, Electa, Milano, pp. 209-211.

34. Per problemi di reperimento dell'immagine, si rimanda a A. Bonito Oliva, op. cit., p. 241.

35. Per un confronto iconografico riguardo alle teorie che collegano sumeri a forme di vita aliene si rimanda a I. Tomassoni, op. cit., p. 1996.

\section{ABSTRACTS}

Gino de Dominicis è sempre stato ossessionato dalla presenza di esseri ultraterreni che potessero influenzare la vita delle persone sulla terra. A partire dagli anni Ottanta le sue tele si popolano di strani riferimenti simbolici ad antiche culture artistiche, come quella sumera. Lungo tutto l'arco della sua produzione artistica Gino de Dominicis ha sempre cercato di contestare il dogma scientifico per avvallare una verità personale; uno dei suoi più curiosi tentativi è stato quello di ricercare l'immortalità nella realtà fisica quotidiana. Il presente saggio, oltre a inquadrarlo all'interno del contesto artistico italiano, propone un'inedita lettura dell'influenza delle teorie pseudoscientifiche nei lavori dell'artista anconetano.

\section{INDEX}

Mots-clés: Gino de Dominicis - Pittura - Extraterrestre - Immortalità - Sumeri 